\title{
Über Eigenschaften neuer Substrate zur Bestimmung von Pankreasenzymen
}

\author{
Von J. G. Meyeri-Bertenrath und H. Kaffarnik \\ Aus der Medizinischen Poliklinik der Universität Marburg/L. (Direktor Prof. Dr. G. W. Löbr)
}

(Eingegangen am 29. Juli 1968)

\section{Herrn Prof. Dr. H.-E. Bock qum 65. Geburtstag gewidmet}

\begin{abstract}
Mit der Synthese der bisher unbekannten Di-Fettsäure-Ester des Fluoresceins (gerader C-Atom-Zahlen von $\mathrm{C}_{8}$ bis $\mathrm{C}_{16}$ ) sind Substanzen verfügbar geworden, die in ihrem physiko-chemischen Verhalten den Eigenschaften natürlicher Lipasesubstrate nahekommen. Die Ester sind nur schwer wasserlöslich und in intakter Form nicht resorbierbar. In Abhängigkeit von der Kettenlänge der Estersäuren werden sie durch Lipase ${ }^{1}$ ), zum Teil auch durch $\alpha$-Chymotrypsin, mit unterschiedlichen Geschwindigkeiten verseift. Das dabei freigesetzte Fluorescein wird resorbiert und bei hoher Clearance durch die Nieren wieder eliminiert. Da die Substrate gegenüber den übrigen Enzymen und Milieubedingungen des Gastro-Intestinal-Traktes stabil sind, ist die Menge des in einem definierten Zeitraum nach oraler Applikation eines Farbstoffesters ausgeschiedenen Fluoresceins ein Kriterium für die im Duodenum vorliegende Pankreasenzymkonzentration.
\end{abstract}

\section{The properties of new substrates for the determination of pancreas enz ymes}

The synthesis of the hitherto unknown fluorescein-carboxylic acid-diesters with straight chain $\left(\mathrm{C}_{\mathbf{8}}-\mathrm{C}_{16}\right)$ fatty acids has made substances available, whose physico-chemical properties closely resemble those of natural lipase substrates. The esters have very low water-solubility, and the intact substances are not absorbed. Depending on the chain length of the constituent acids, the esters are saponified at varying rates by lipase, and some of them by $\alpha$-chymotrypsin. The released fluorescein is absorbed and eliminated by the kidneys with a high clearance. Since the substrates are stable to other enzymes and environments in the gastro-intestinal tract, the amount of fluorescein excreted in a defined period following the oral application of a fluorescein ester is a criterion for the concentration of pancreatic enzymes in the duodenum.

Die Diagnostik von Insuffizienzen der exokrinen Pankreasfunktion hat bisher auf einfache und zuverlässige Laboratoriumsmethoden verzichten müssen. Die Erfassung der sezernierten Enzymmengen durch die Untersuchung des mittels einer Duodenalsonde gewonnenen Materials ist ein schwieriges Verfahren, dessen Bedeutung vor allem durch den experimentellen und zeitlichen Aufwand begrenzt geblieben ist. Die Bestimmung der $\alpha$-Amylase im Serum oder Urin dagegen ist methodisch zwar einfach, sie wird jedoch in der Aussagekraft durch vielfach unspezifische Befunde eingeengt.

Mit der Synthese von nicht resorbierbaren Farbstoffestern, aus denen nach oraler Gabe durch die Einwirkung der Pankreasenzyme Lipase und Chymotrypsin die gefärbte Komponente freigesetzt und resorbiert wird, ist eine erhebliche Vereinfachung der Pankreasdiagnostik möglich geworden. Es hat sich gezeigt, daß die im Urin ausgeschiedene Fluoresceinmenge der Enzymkonzentration im Duodenum proportional ist und somit eine quantitative Abstufung der exokrinen Pankreasfunktion erlaubt (1). Der vorliegende Beitrag befaßt sich mit einer Darlegung von physikalischchemischen Eigenschaften der Testsubstanzen und ihrem Verhalten unter verschiedenen in-vitro-Bedingungen, nachdem über Synthese und Analysendaten der Ester bereits an anderer Stelle berichtet worden ist (2).

\section{Methodik}

Die Inkubation der Substrate erfolgte ausnahmslos in Tris- $\mathrm{HCl}$ Puffer, $0,1 \mathrm{M}, \mathrm{pH} 8,0$ und einer Temperatur von $37^{\circ} \pm 0,1 \%$ (Colora-Ultrathermostat). Die Ester wurden zunächst in Åthylen-

1) Enzyme: Lipase: Glycerol-Ester Hydrolase (EC 3.1.1.3.). $\alpha$-Chymotrypsin $=(E C$ 3.4.4.5). $\alpha$-Amylase $=\alpha$-1,4-Glucan 4-Glucanohydrolase (EC 3.2.1.1). Alkalische Phosphatase = Orthophosphorsäure Moneester-Phosphohydrolase (EC 3.1.3.1). glykolmonomethyläther (Methylcellosolve) zu Stammlösungen von $1 \mathrm{~mm}, 5 \mathrm{~mm}$ oder $10 \mathrm{~mm}$ (niedere Homologe) gelöst, die auch bei Raumtemperatur über Wochen stabil waren. Aus diesen Lösungen wurden die zur gewünschten Verdünnung notwendigen Volumina (Konstriktionspipetten) in Meßkölbchen vorgelegt, zur Einstellung einer in allen Verdünnungen identischen Konzentration an Methylcellosolve mit diesem Lösungsmittel nachkorrigiert und mit Puffer auf $4 / 5$ jenes Volumens aufgefüllt, das zur Erreichung der Endkonzentration notwendig war. Mit je $1 \mathrm{~m} l$ Lösung wurden Mikroküvetten in einem temperierbaren Küvettenhalter mit Wechselautomatik (Eppendorf-Photometer 1100M) beschickt. Nach dem Temperaturausgleich wurde nunmehr von der Enzymlösung, die im gleichen Puffer in einer relativ zur Endkonzentration um den Faktor 5 höheren Konzentration stets unmittelbar vor Versuchsbeginn bereitet worden war, jeweils $0,25 \mathrm{~m} l$ hinzupipettiert, gut gemischt und der Anstieg der Extinktion bei $492 \mathrm{~nm}$ durch die Fluoresceinfreisetzung mit dem Kompensationsschreiber (Eppendorf 4412, System Philips) aufgezeichnet. Die Reaktionsgeschwindigkeit wurde im allgemeinen als $\frac{\Delta \mathrm{E}}{\Delta \mathrm{t}}=\operatorname{tg} \alpha$ ausgedrückt, gemessen in den ersten $5 \mathrm{Min}$. nach Start der Reaktion. Soweit nicht anders angegeben, wurden Enzympräparationen der Firmen Serva, Heidelberg ( $\alpha$-Chymotrypsin, Lipase aus Aspergillus und intestinale alkalische Phosphatase) oder Koch-LightLaboratories Ltd. (verschiedene Chymotrypsine) eingesetzt.

\section{Ergebnisse}

\section{Eigenschaften der Testsubstanzen}

Die Farbigkeit des Fluoresceins wird durch die chinoide Gruppierung (II), die dưrch die Öffnung des Lactonringes (I) möglich wird, erklärt. Da mit der Veresterung beider phenolischer Hydroxylgruppen (III) die Ausbildung des chinoiden Chromophors nicht mehr möglich ist, resultieren vollkommen farblose Ester. Diese Tatsache ist für die in-vitro-Untersuchungen außerordentlich nützlich, da erst als direktes $\mathrm{Ma} \beta$ einer ablaufenden enzymatischen oder alkalischen Verseifung die Färbung (II) wieder auftritt, die durch Absorptionsoder Fluoreszenzphotometrie mit hoher Empfindlichkeit meßbar ist (10). 


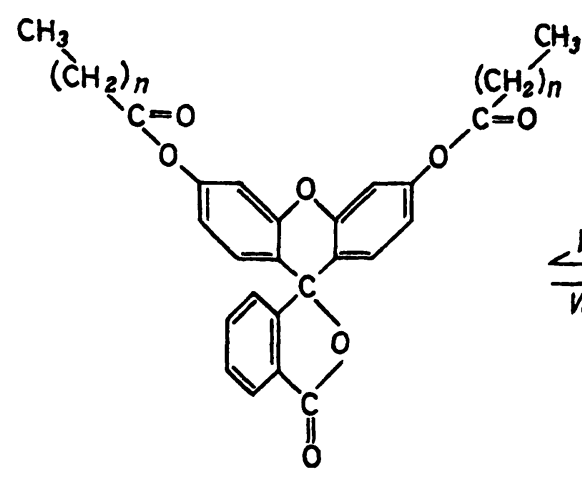

III forblos
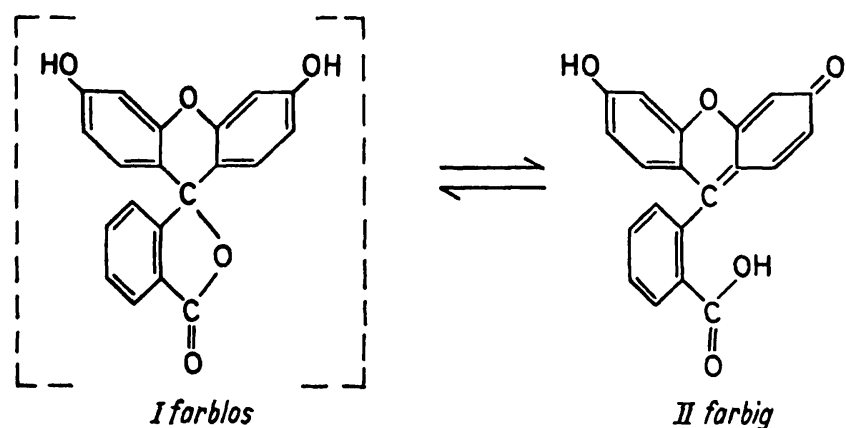

II forbig
In unseren Versuchen wurde auf die Ausnutzung der Fluoreszenz zunächst verzichtet, da unter den gewählten Bedingungen die Absorptionsmessungen vollkommen ausreichend waren. Wie aus Abbildung 1 hervorgeht, sind Absorptionsmaximum $\left(\lambda_{\max }\right)$ und molarer Extinktionskoeffizient $(\varepsilon)$ des Fluoresceins stark pH-abhängig. Für die in alkalischem Milieu durchgeführten Inkubationen war die Verschiebung der $\lambda_{\max }$ auf $492 \mathrm{~nm}$ mit einem Anstieg der molaren Extinktion auf $0,7 \cdot 10^{5} \mathrm{~cm}^{2} / \mathrm{Mol}$ und somit einer Erhöhung der Empfindlichkeit verbunden. Der molare Extinktionskoeffizient für Fluorescein in alkalischer Lösung errechnet sich demnach mit $\varepsilon_{492 \mathrm{~nm}}=70 \cdot 10^{6} \mathrm{~cm}^{2} / \mathrm{Mol}$, liegt also etwa um den Faktor 11 höher als der des $\mathrm{NADH}\left(\varepsilon_{340 \mathrm{~nm}}=6,2 \cdot 10^{6} \mathrm{~cm}^{2} / \mathrm{Mol}\right)$.

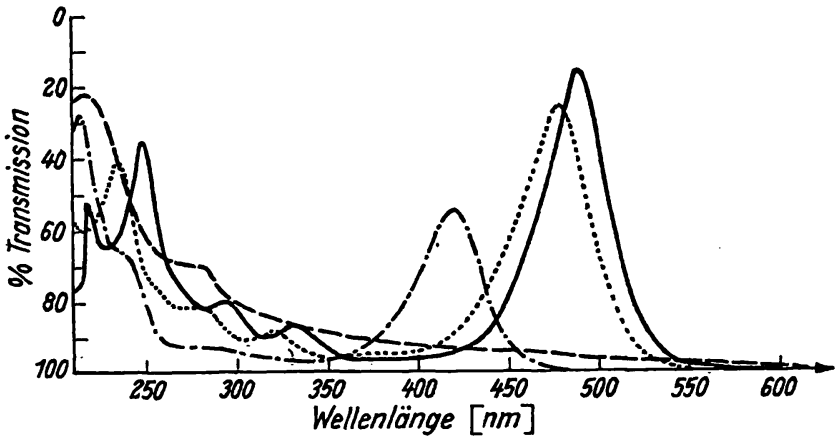

Abb. 1

Absorptionsspektren des Fluoresceins bei verschiedenem $\mathrm{pH}$ (pH $7 \ldots$ Absorptionsspektren des Fluorescelns bei verschiedenem pH (pH 7...

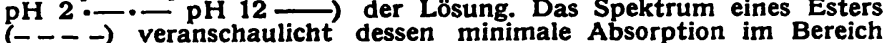
des sichtbaren Lichts

Für in-vitro-Inkubationen bedeutet die geringe Wasserlöslichkeit der Fluoresceinester eine experimentelle Schwierigkeit, die nur durch Verwendung organischer Lösungsmittel zu umgehen war. Als geeignet erwiesen sich sowohl Tween 80 als auch Äthylenglykolmonomethyläther (= Methylcellosolve). Wegen der besseren Löslichkeit vor allem der höheren Homologen in Methylcellosolve, die die Herstellung von $5 \mathrm{~mm}$ Stammlösungen erlaubte, wurde diesem Lösungsmittel der Vorzug gegeben. Die MethylcellosolveKonzentration in den Inkubationsansätzen wurde unabhängig von der Substratkonzentration auf konstante Werte nachkorrigiert und lag entweder bei 0,8 oder $1 \%$. Eine Beeinflussung der enzymatischen Reaktionen liegt bei diesen Bedingungen nicht vor. Die Fluoresceinester bleiben nach der Verdünnung der Methylcellosolve-
Stammlösungen mit Tris-HCl-Puffer in Abhängigkeit von der Kettenlänge ihrer Fettsäuren in Lösung. Die Grenzkonzentrationen (beginnende Trübung: $\mathrm{E}_{492} \geqq$ $0,1)$ liegen für die $C_{6}$ - und $C_{8}$-Ester bei $60 \mu \mathrm{M}$, für die nächst höheren Homologen $\left(C_{10}-C_{14}\right)$ bei 50 und für das $\mathrm{C}_{16}$-Derivat bei $40 \mu \mathrm{M}$.

Sowohl in Methylcellosolve als auch in saurem und neutralem wäßrigen Milieu sind die Fluoresceinester bei Raumtemperatur stabil. In alkalischer Lösung tritt erst bei $\mathrm{pH} 9,8 \mathrm{im}$ Verlaufe mehrerer Tage eine geringe Hydrolyse ein, die jedoch mit steigendem $\mathrm{pH}$ rasch zunimmt.

Die enzymatische Hydrolyse der FluoresceinEster

Lipase aus Rbizopus arrbizus, die in hochgereinigter Form mit einer Aktivität von $3800 \mathrm{IE} / \mathrm{mg}$ vorlag, spaltet die Substrate in Abhängigkeit von der Kettenlänge der Estersäuren (Abb. 4). Die Aufzeichnung der Hydrolyse bei steigender Substratkonzentration (Abb. 2)

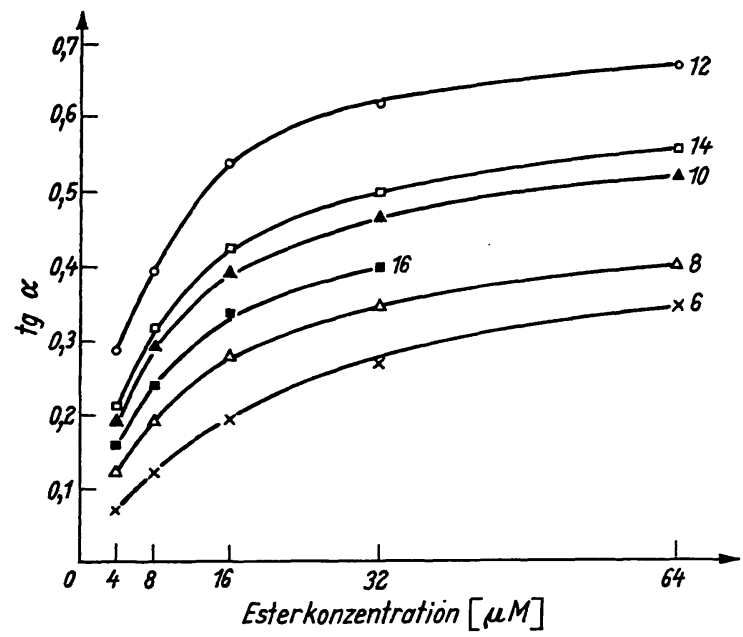

Abb. 2

Abhängigkeit der enzymatischen Esterverseifung von den Kettenlängen der Estersäuren (Ziffern an den Kurven = Anzahl der C Atome) und der Esterkonzentration durch Lipase aus Rhizopus arrhizus $\left(2,5 \mu \mathrm{g} / \mathrm{ml}\right.$, pH $\left.8,0,37^{\circ}\right)$. Auf der Ordinate ist der tg $\alpha$ als Ausdruck der Reaktionsgeschwindigkeit abgetragen

läßt erkennen, daß die Ester sowohl in gelöster als auch in emulgierter Form reagieren, ohne beim Wechsel des Aggregatzustandes einen unter den gegebenen Versuchsbedingungen erkennbaren Sprung der Reaktionsgeschwindigkeit $\mathrm{zu}$ induzieren, wie es für die Einwirkung von Pankreaslipase auf Triglyceride be- 
schrieben wird (3). Die Reaktion folgt der.MichaelisMenten-Beziehung. Nach kinetischen Messungen der Reaktionsgeschwindigkeiten sind die Michaelis-Konstanten (Tab. 1) aus Lineweaver-Burk-Diagrammen (Abb. 3) graphisch ermittelt worden.

Tab. 1

Abhängigkeit der Michaelis-Konstanten ( $\mathrm{K}_{m}$ in $\left.10^{-5} \mathrm{Mol} / \mathrm{l}\right)$ von der Länge der Fettsäuren der Fluoresceindiester. Die Inkubationen erfolgten in Tris-HCl-Puffer $(0,1 \mathrm{M})$ bei $\mathrm{pH}=8,0$ und $37^{\circ}$

\begin{tabular}{|c|c|c|c|c|c|c|}
\hline Enzym & $\mathrm{C}_{0}$ & $\begin{array}{c}\text { Ketten } \\
\mathbf{C}_{8}\end{array}$ & $\begin{array}{r}\text { länge } \\
\mathrm{C}_{10} \\
\end{array}$ & $\begin{array}{c}\text { er Est } \\
\mathrm{C}_{12} \\
\end{array}$ & $\begin{array}{c}\text { rsäuren } \\
\mathrm{C}_{\mathbf{1 4}} \\
\end{array}$ & $\mathrm{C}_{10}$ \\
\hline \multirow{2}{*}{$\begin{array}{l}\text { Lipase aus Rhizopus arrhi- } \\
\text { zus, } 2,5 \mu \mathrm{g} / \mathrm{ml} \\
\text { Lipase aus Aspergillus ory- } \\
\text { zae, } 100 \mu \mathrm{g} / \mathrm{ml}\end{array}$} & 2,35 & 1,13 & 0,81 & 0,65 & 0,73 & 0,89 \\
\hline & 1,75 & 1,67 & 1,52 & 1,33 & 1,47 & 1,58 \\
\hline
\end{tabular}

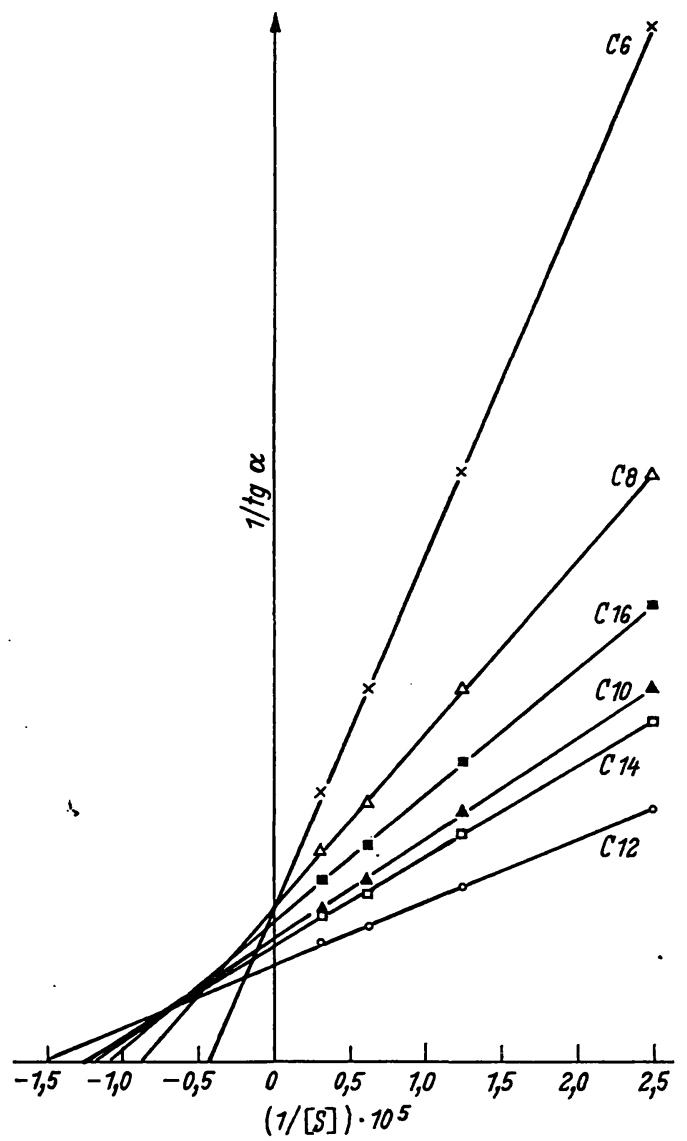

Abb. 3

Lineweaver-Burk-Diagramme der in Abbildung 2 dargestellten $\mathrm{Be}$ ziehungen. Die abgelesenen Michaelis-Konstanten sind in Tabelle 1 wiedergegeben

Es ist bemerkenswert, daß die Abhāngigkeit der Esterverseifung von der Kettenlänge der Fettsäureester ein Optimum durchläuft (Abb. 4). Der maximal lipaseempfindliche $\mathrm{C}_{12}$-Ester ist daher zur Bestimmung der Hydrolysegeschwindigkeit bei verschiedenen Enzymkonzentrationen eingesetzt worden (Abb. 5).

Lipase aus Aspergillus verhält sich grundsätzlich ähnlich wie das Rbizopus-Präparat. Die Michaelis-Konstanten (Tab. 1) sind wieder aus Lineweaver-Burk-Diagrammen abgelesen worden

Mit $\alpha$-Chymotrypsin lassen sich praktisch nur der Capronund Caprylsäureester in nennenswertem Umfange verseifen, während eine Hydrolyse der höhermolekularen Ester in den ersten Minuten kaum meßbar ist (Abb. 4, 7).

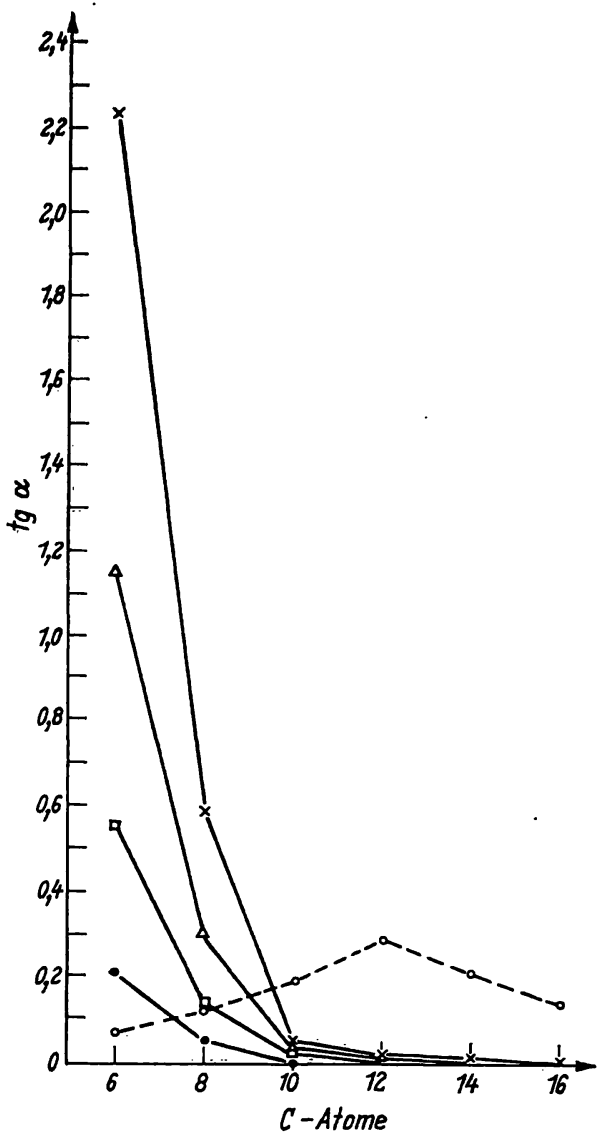

Abb. 4

Esterverseifung durch $\alpha$-Chymotrypsin $\left(0,1 \mathrm{mg} / \mathrm{ml}, \mathrm{pH} 8,0,37^{\circ}\right)$ in Abhängigkeit von der Molekülgröße des Substrats. Die Esterkonzentrationen betrugen $40(x), 20(\Delta) 10(ㄱ)$ und 5 (॰) $\mu \mathrm{M}$. Zum Vergleich ist die Empfindichkeit der Ester $(5 \mu \mathrm{M})$ gegenüber Lipase aus Rhizopus arrhizus (vgl. Abb. 2) mit aufgetragen worden (---)

Ähnlich verhalten sich, wenn auch mit geringerer Aktivität, $\beta$-, $\gamma$-, $\delta$ - und $\pi$-Chymotrypsin.

Pankreatin mit einer Aktivität von $25 \mathrm{IE} / \mathrm{mg}$ zeigt einen atypischen Reaktionsverlauf (Abb. 6), der eine Überlagerung zweier Enzymaktivitäten vermuten läßt. Geht man von der bevorzugten Spaltung der langkettigen Ester durch Lipase aus, so resultiert eine Fremdaktivität mit maximalem Angriff der kurzkettigen

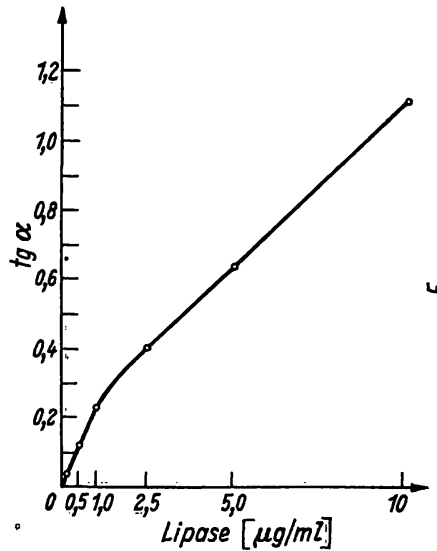

Abb. 5

Abhängigkeit der Esterverseifung $\left(\mathrm{C}_{12}\right.$-Ester, $50 \mu \mathrm{M}$, pH 8,0, $37^{\circ}$ ) von der Lipasekonzentration (Rhizopus arrhizus)

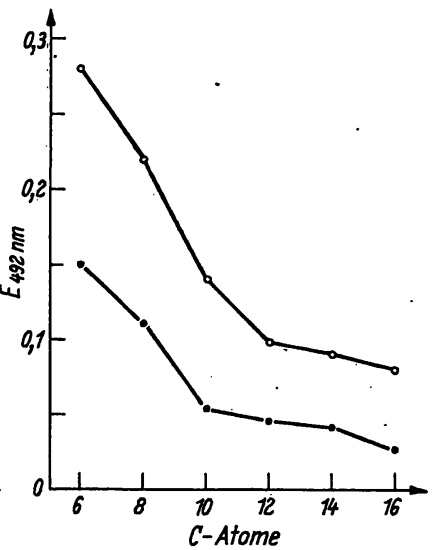

Abb. 6

Esterverseifung (vgl. Abb. 4) durch Pankreatin $(0,025 \mathrm{mg} / \mathrm{ml})$, $\mathrm{pH}=8,0,37^{\circ}$ ) bei verschiedenen Substratkonzentrationen: $\circ-0=50 \mu \mathrm{M},-\frac{-10}{=} 10 \mathrm{M}$. Als Ausdruck der eingetretenen Hydrolyse wurde nach einer Inkubationszeit von 30 Min. die Extinktion bei $492 \mathrm{~nm}$ bestimint 


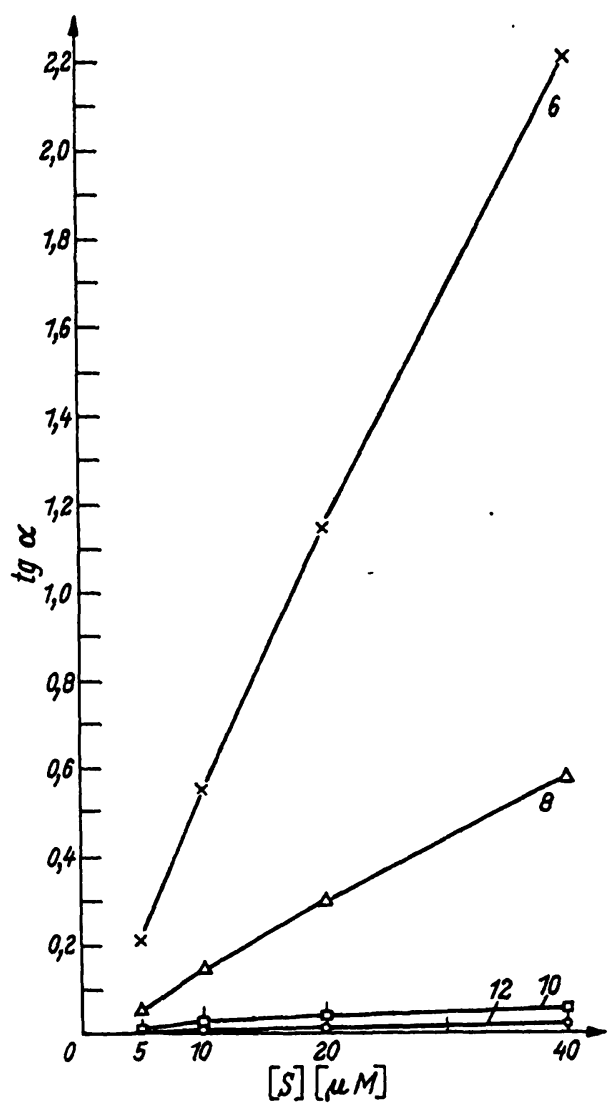

Abb. 7

Abhängigkeit der Hydrolyse der $C_{0}-$ bis $C_{13}$-Ester des Fluoresceins durch $\alpha$-Chymotrypsin $(0,1 \mathrm{mg} / \mathrm{ml})$ von der Substratkonzentration

Substrate. Wie oben schon dargestellt wurde (Abb. 4), ist ein solches Verhalten für das $\alpha$-Cbymotrypsin charakteristisch, das demnach Bestandteil der PankreatinPräparation sein dürfte. Versuche mit Protease-Inhibitoren, die zur experimentellen Fundierung dieser Vermutung unternommen worden sind, erbrachten wegen zu geringer Spezifität der Hemmung keine verwertbaren Aussagen. Alkalische Plosphatase zeigt überraschenderweise ebenfalls hydrolytische Aktivität, die jedoch auf die Fluoresceinester mit kurzkettigen Fettsäuren (bis zum $\mathrm{C}_{8}$-Derivat) beschränkt ist. Diese Aktivität ist durch Protease-Inhibitoren (Trasylol, Diphenylcarbamylchlorid) zu beeinflussen und daher wahrscheinlich durch Chymotrypsinspuren des Präparates bedingt. Ein negativer Einfluß der Hemmstoffe auf die Aktivität der alkalischen Phosphatase konnte im kinetischen Test (Substrat: $p$-Nitrophenylphosphat) ausgeschlossen werden.

\section{Diskussion}

Der ersten Publikation aus dem Jahre 1912 über die Darstellung des Diacetylfluoresceins (4) folgten erst kürzlich Mitteilungen über einige kurzkettige Homologe $(5,6)$, die zu thermodynamischen Studien eingesetzt wurden, ohne jedoch klinische Fragestellungen zu berühren.

Die Eignung von Enzymsubstraten zur Pankreasdiagnostik setzt eine ausreichende Spezifität voraus. Durch den Nachweis der Stabilität der Fluoresceinester gegenüber den Milieubedingungen des Gastro-Intestinal-Traktes bei fehlender Sekretion aus dem Pankreas (1) ist eine eingetretene Hydrolyse der Ester als spezifischer Hinweis auf die Ausschüttung von enzymhaltigem Pankreassekret zu betrachten. Bei den niedermolekularen Substraten, vor allem dem Fluoresceindicapronsäureester, überwiegt die Aktivität des oChymotrypsins die Hydrolyse durch Lipase. Diese Esteraseaktivität des Chymotrypsins ist vergleichbar der Spaltung des p-Nitrophenylacetats (7), das als Indikatorsubstanz zur Bestimmung dieses Enzyms eingesetzt wird und als Phenolester dem gleichen Substanztyp angehört, wie er durch die Veresterung der phenolischen Hydroxylfunktionen des Fluoresceins geschaffen wurde. Die hier mit steigender Kettenlänge der Estersäuren rasch abfallende hydrolytische Aktivität des Enzyms ist mit der Annahme zunehmender sterischer Hinderung zufriedenstellend interpretierbar, zumal wir über die Spaltungskinetik durch $\alpha$-Chymotrypsin wissen, $\mathrm{da} \beta$ dic Hydrolyse in drei reversiblen, konsekutiven Schritten am Enzym abläuft (8):

\section{Bildung des Enzym-Substrat-Komplexes,}

2. Intramolekulare Umwandlung zum acylierten Enzym unter Freisetzung der alkoholischen Komponente des Substrates,

3. Hydrolyse des Acylenzyms zu Säure und aktivem Enzym.

Die Acylierung des reaktiven Serinhydroxyls (Schritt 2) kann, wie in Untersuchungen mit bifunktionellen Substanzen verschiedenen Abstandes der reaktiven Gruppen ausgemessen wurde (9), nur bei einer geeigneten räumlichen Beziehung des Substratmoleküls zum Serin- und Histidinrest des aktiven Zentrums einerseits und dem Substratbindungszentrum andererseits erfolgen. Es ist offensichtlich, daß die stark hydrophoben aliphatischen Reste mit zunehmder Kettenlänge diese Voraussetzungen schrittweise aufheben.

Uber die Lipasen verschiedener Provenienz liegen bislang keine vergleichbaren Informationen vor. Für hochgereinigte Pankreaslipase wird eine Entfaltung maximaler lipolytischer Aktivität nur an emulgierten Substraten beschrieben (3), insbesondere an Triglyceriden. Diese Beobachtung konnte unter den hier gewählten Versuchsbedingungen für die eingesetzten Pilzlipasen nicht wiederholt werden; wie oben geschildert, ist eine Unstetigkeit des Reaktionsablaufs bei eintretender Emulgierung des Substrats nicht festgestellt worden. Bemerkenswert ist der Befund, da $\beta$ die Hydrolyserate in Abhängigkeit von der Kettenlänge der Estersäuren ein Optimum durchläuft. Auch damit wird deutlich, daß eine beginnende Emulgierung, d. h. die als Funktion der Fettsäurelänge zu verstehende kritische Konzentration der Ester, nicht als zusätzlicher Parameter in die Spaltungskinetik eingeht: Einerseits wird trotz höherer Anteile an emulgiertem Substrat der $\mathrm{C}_{16}$-Ester langsamer verseift als der $\mathrm{C}_{12}$-Ester. Der $\mathrm{C}_{8}$-Ester hingegen unterliegt ebenfalls einer relativ 
langsamen Hydrolyse, obwohl die emulgierten Anteile hier geringer sind als beim $\mathrm{C}_{12}$-Ester gleicher Konzentration.

Für die Verwendung zum Pankreasenzymtest bietet sich nach diesen Untersuchungen der Fluoresceindilaurinsäureester vor allem deshalb als optimale Substanz an, weil seine Spaltung praktisch vollkommen durch Lipase katalysiert wird und den Test damit zu einem spezifischen Lipasetest werden läßt. Bei Einsatz z. B. des Capronsäureesters dagegen resultiert eine Verseifung, die zwar überwiegend durch Chymotrypsin bedingt wird, an der jedoch die Lipase noch einen merklichen Anteil hat. Die sehr viel schnellere Hydrolyse des $\mathrm{C}_{6}$-Esters erfordert darüber hinaus die Applikation größerer Substratmengen und bedeutet daher für die Praxis des Tests einen methodischen Nachteil.

Für die Unterstützung der Untersuchungen danken wir der Deutschen Forschungsgemeinschaft.

Für die freundliche Utberlassung von Lipase aus Rbizopus arrbizus und Pankreatin danken wir Herrn Dr. F. H. Schmidt (Fa. Boehringer, Mannheim).

\section{Literatur}

1. Kaffarnik, H. und J. G. Meyer-Bertenrath, Klin. Wschr. (im Druck). - 2. MeYer-Bertenrati, J. G., Hoppe-Seyler's Z. physiol. Chem. 349, 728 (1968). - 3. Sarda, L. und P. Desnuelle, Biochim. biophysica Acta Amsterdam 30, 513 (1958). - 4. v. Liebig, H., J. prakt. Chem. 85, 267 (1912). - 5. Guilbault, G. G. und D. N. Kramer, Analytic. Chem. 36, 409 (1964). - 6. Guilbault, G. G. und D. N. KRAmer, Analytic. Biochem. 14, 28
(1966). - 7. Hartley, B. S. und B. A. Krlby, Biochem. J. 50, 672 (1952). - 8. ErLanger, B. F. und W. Cohen, J. Amer. chem. Soc. 85, 348 (1963). - 9. GundLACH, G., Habilitationsschrift Universität Würzburg (1964). - 10. MEYER-BERTENRATH, I. G. und H. Kaffarnik, Hoppe-Seyler's Z. physiol. Chem. 349, 1071 (1968). 